\title{
Enhanced anaerobic digestion of swine manure by the addition of zero-valent iron
}

\author{
Yuan Yang ${ }^{1}$, Jianyu Wang ${ }^{1}$, Yanbo Zhou ${ }^{1,2^{*}}$ \\ ${ }^{1}$ State Environmental Protection Key Laboratory of Environmental Risk Assessment \\ and Control on Chemical Process, East China University of Science and Technology, \\ No. 130 Meilong Road, Xuhui District, Shanghai 200237, China \\ 2 Shanghai Institute of Pollution Control and Ecological Security, No. 1515 \\ Zhongshan Second North Road, Hongkou District, Shanghai 200092, China

\section{Corresponding author} \\ *Yanbo Zhou. Email: zhouyanbo@ecust.edu.cn. Tel./fax:021-64250225. Contact address: State \\ Environmental Protection Key Laboratory of Environmental Risk Assessment and Control on \\ Chemical Process, East China University of Science and Technology, No. 130 Meilong Road, Xuhui \\ District, Shanghai 200237, China
}


Table S1. The material addition of 6 reactors.

\begin{tabular}{cccccc}
\hline Batch No. & $\begin{array}{c}\text { Fresh swine } \\
\text { manure (g) }\end{array}$ & Inocula (g) & $\begin{array}{c}\text { ZVI supplementation } \\
\text { on acidogenic stage }(\mathrm{g})\end{array}$ & $\begin{array}{c}\text { ZVI supplementation on } \\
\text { methanogenic stage }(\mathrm{g})\end{array}$ & $\begin{array}{c}\text { Total ZVI dosage } \\
(\mathrm{g} / \mathrm{L})\end{array}$ \\
\hline R1 & 300 & 200 & 0 & 0 & 0 \\
R2 & 300 & 200 & 0 & 0 & 12 \\
R3 & 300 & 200 & 6 & 3 & 12 \\
R4 & 300 & 200 & 3 & 0 & 0 \\
R5 & 300 & 200 & & 6 & 12 \\
R6 & 300 & 200 & & & \\
\hline
\end{tabular}

${ }^{\text {a }}$ Single-stage anaerobic digestion

Table S2. Parameters of fitting kinetic model equation of R1 and R5.

\begin{tabular}{ccccc}
\hline Reactors & $\mathrm{B}_{0}\left(\mathrm{~mL} / \mathrm{g} \cdot \mathrm{VS}_{\text {fed }}\right)$ & $\mathrm{R}_{\mathrm{m}}\left(\mathrm{mL} / \mathrm{g} \cdot \mathrm{VS}_{\text {fed }} \cdot \mathrm{d}\right)$ & $\lambda(\mathrm{d})$ & $\mathrm{R}^{2}$ \\
\hline $\mathrm{R} 1$ & $157.82 \pm 1.85$ & $15.86 \pm 0.32$ & $4.19 \pm 0.09$ & 0.9990 \\
$\mathrm{R} 5$ & $149.87 \pm 2.86$ & $13.34 \pm 0.36$ & $3.90 \pm 0.13$ & 0.9983 \\
\hline
\end{tabular}

Table S3. Recovery of zero-valent iron

Reactors $\quad$ Mass of total ZVI (g) $\quad$ Mass of recovery ZVI (g) $\quad$ Recovery rate (\%)




\begin{tabular}{cccc}
\hline R2 & 6 & $4.24 \pm 0.35$ & $70.67 \pm 8.25$ \\
R3 & 6 & $3.84 \pm 0.30$ & $64.00 \pm 7.81$ \\
R4 & 6 & $3.95 \pm 0.27$ & $65.83 \pm 6.84$ \\
R6 & 6 & $4.17 \pm 0.23$ & $69.50 \pm 5.52$ \\
\hline
\end{tabular}




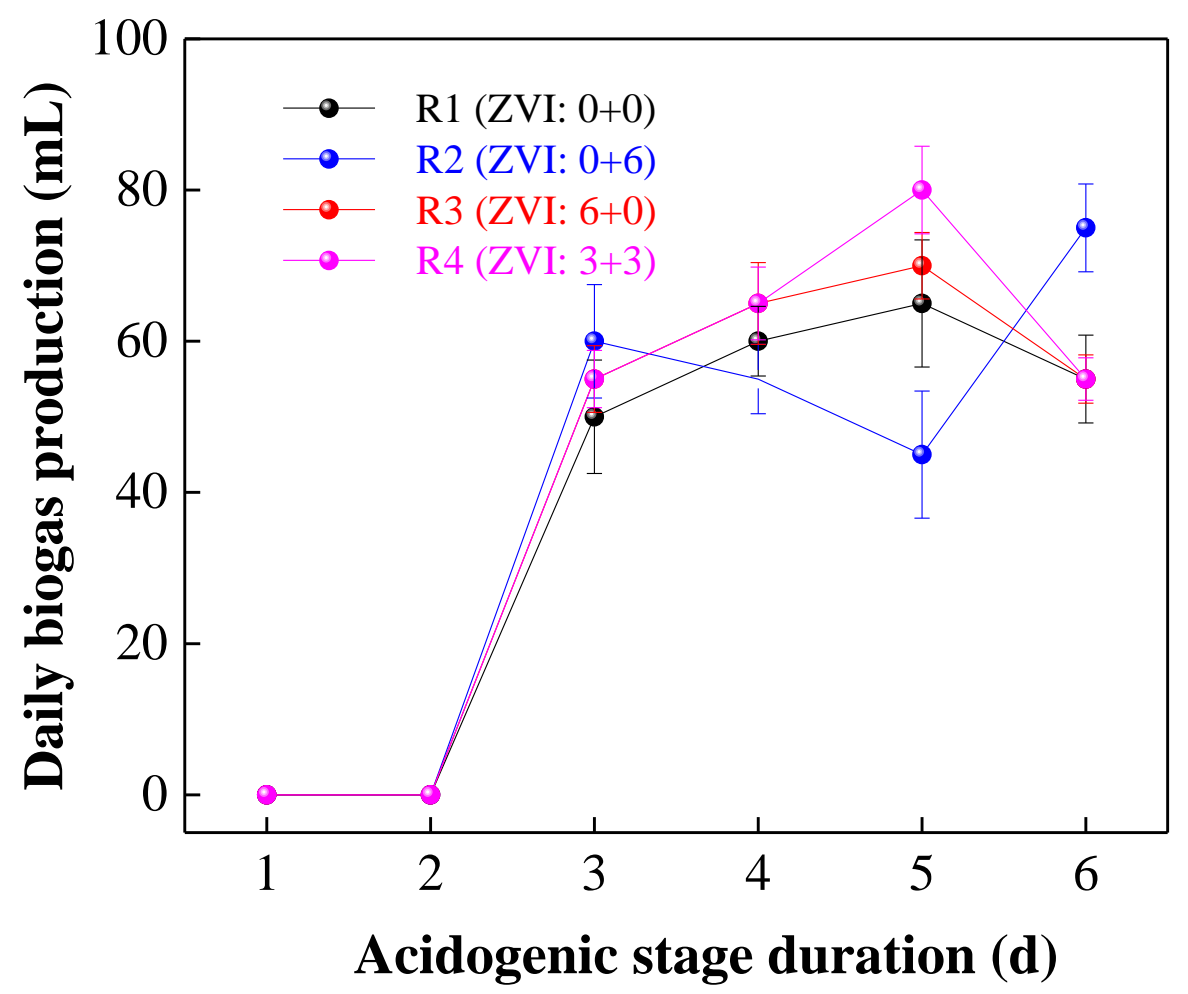

Fig. S1. Profiles of biogas production during acidogenic stage for R1-R4. 


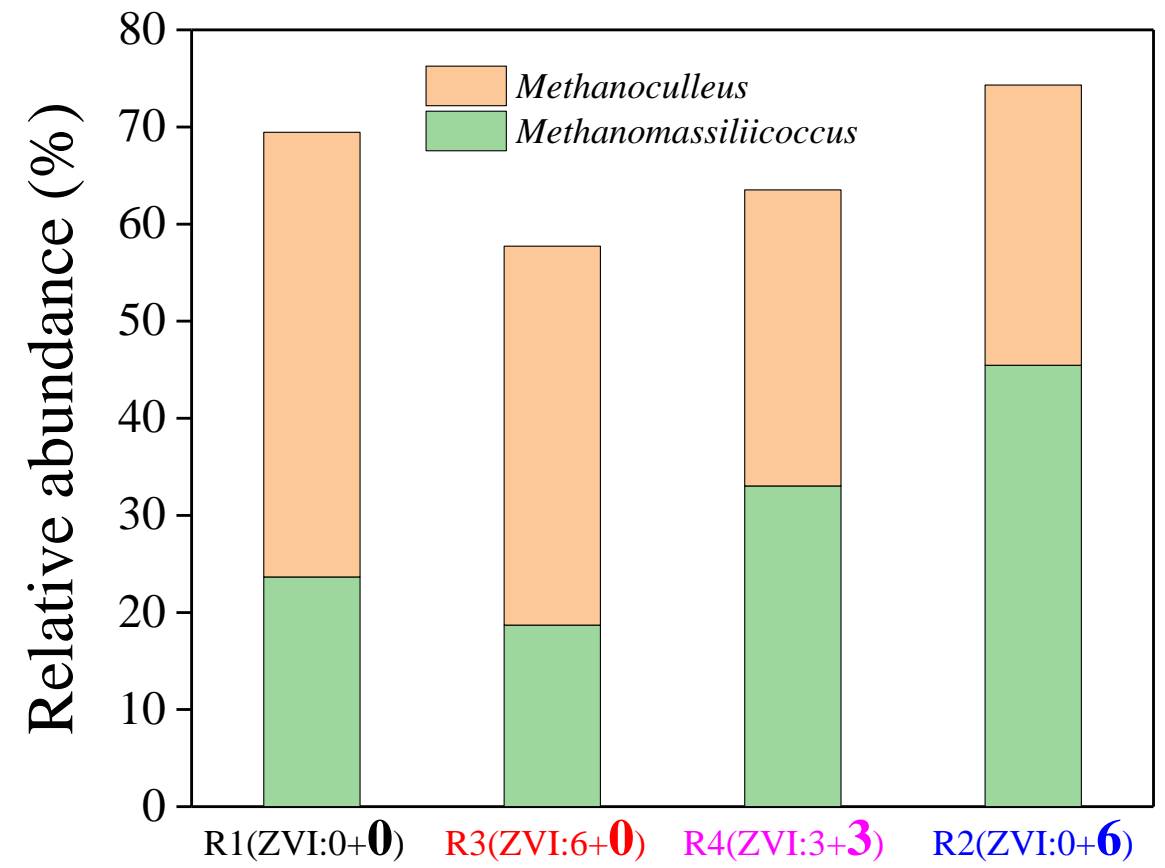

Fig. S2. The relative abundance relationships between Methanoculleus or Methanomassiliicoccus and ZVI dosage on methanogenic stage. 